\title{
Evaluación Cualitativa de un Sistema de Monitoreo de la Convivencia Escolar
}

\author{
Claudia Carrasco Aguilar'; https://orcid.org/0000-0002-8768-2440 \\ Verónica López; https://orcid.org/0000-0001-7405-3859 \\ Paula Ascorra²; https://orcid.org/0000-0001-9449-8273 \\ M. Angeles Bilbao3; https://orcid.org/0000-0002-5984-4908 \\ Simoné Olmos²; https://orcid.org/0000-0002-0729-3972
}

\section{Resumen}

En el marco de un proyecto para el diseño, desarrollo y validación de un sistema de monitoreo de la convivencia escolar, el presente estudio evalúa dicho sistema a partir de los significados construidos por sus usuarios, con el fin de mejorar acciones futuras. Se realizaron 17 entrevistas grupales en15 escuelas, más una entrevista en profundidad a la Coordinadora Comunal de Convivencia Escolar. Resultados: Las escuelas participantes señalan que el sistema de monitoreo destaca por la entrega de información válida, pues permite la toma de decisiones basadas en evidencia logrando complejizar la mirada que las escuelas tienen de sí mismas. Discusiones: Las escuelas colocan en el centro de la mejora escolar a la propia escuela, y destacanque la efectividad sólo es posible si la priorización de necesidades surge del propio colegio.

Palabras clave: Evaluación; violencia; medio escolar.

\section{Qualitative evaluation of a System for Monitoring School Climate}

\begin{abstract}
As part of a project in which we designed, developed, and validated a monitoring system of school climate, the present study evaluates the system based on the meanings constructed by its users, in order to improve future actions. 17 group interviews in 15 schools, plus an in-depth interview to the Community Coordinator School Coexistence were performed. Results: Participating schools indicate that the highlight of the monitoring system is the delivery of valid information that allow evidence-based decision making processes. This in turn allows schools to construe a more complex view of themselves. Discussions: Schools place, in the center of school improvement, the school itself, and emphasize that effectiveness is only possible if the prioritization of needs arises from the school itself.
\end{abstract}

Keywords: Evaluation; violence; school environment.

\section{Avaliação Qualitativa de um Sistema de Monitoração da Convivência Escolar}

\section{Resumo}

No marco de um projeto para a concepção, desenvolvimento e validação de um sistema de monitoração da convivência escolar, o presente estudo avalia tal sistema a partir dos significados construídos por seus usuários, com o fim de melhorar ações futuras. Foram realizadas 17 entrevistas grupais em15 escolas, mais uma entrevista em profundidade à Coordenadora Comunal de Convivência Escolar. Resultados: As escolas participantes relatam que o sistema de monitoração destaca pela entrega de informação válida, pois permite a tomada de decisões baseadas em evidência logrando problematizar a olhada que as escolas têm de si mesmas. Discussões: As escolas colocam no centro da melhora escolar a própria escola e destacam que a efetividade só é possível se a priorização de necessidades surge do próprio colégio.

Palavras-chave: Avaliação; violência; ambiente escolar.

1 Universidad de Playa Ancha, Región de Valparaíso - Chile; claudia.carrasco@upla.cl

2 Pontificia Universidad Católica de Valparaíso, Centro de Investigación para una Educación Inclusiva, Región de Valparaíso - Chile; veronica. lopez@pucv.cl; paula.ascorra@pucv.cl; simone.olmos@gmail.com

3 Universidad Alberto Hurtado. Santiago, Chile; bilbao.angeles@gmail.com. 


\section{Introducción}

Estudios nacionales e internacionales han demostrado que el clima y convivencia escolar,inciden en los resultados de aprendizaje de los estudiantes (Espinoza, 2006; Giraldo \& Mera, 2014; López \& cols., 2012; Valdés, Treviño, Acevedo, Castro, Carrillo, Costilla, Bogoya, \& Pardo, 2008), a la vez que constituyen parte importante de los procesos formativos que se originan en la escuela, como la convivencia social y ciudadana (Abad, 2002; Magendzo, 2007). La valoración del clima escolar, ha sido interpretadapor diversos teóricos del mejoramiento escolar como un componente de la calidad educativa, impactando en el desarrollo potencial de niños y niñas (Bender Sebring \& Montgomery, 2014; Bolívar, 2010; Bolívar, López, \& Murillo, 2013). Con este fin, en Chile, la convivencia escolar ha sido considerada en las políticas públicas en distintos niveles, como la sala de clases, la Política Nacional (Mineduc, 2003b, 2011, 2015), así como en la elaboración y difusión de estándares indicativos de desempeño para los establecimientos y sus sostenedores (Mineduc, 2014).

Paradójicamente, estas iniciativas han implicado para las escuelas la presión a mejorar en convivencia escolar, con escasos apoyos institucionales, y aunque se han incorporados componentes de clima y convivencia escolar en las mediciones nacionales, no constituyen más del $33 \%$ de los resultados informados (Agencia de Calidad de la Educación, 2015) y su devolución a las escuelas es demasiado general, dificultando orientar decisiones políticas internas.

\section{Monitoreo de la convivencia escolar}

Los datos internacionales sugieren de manera consistente que los programas de mejoramiento más exitosos han sido adecuados para adaptarse a una escuela específica e involucrar a todos los componentes en el ambiente escolar (Astor, Benbenishty, \& Meyer, 2004; Benbenishty \& Astor, 2005; Furlong, Greif, Bates, Whipple, Jimenez, \& Morrison, 2005; Justice, 2006), combinando técnicas de monitoreo y mapeo (Astor, Benbenishty, \& Meyer, 2004), con un enfoque integral y multinivel (Pérez, Astudillo, Varela, \& Lecannelier, 2013). Autores como Van Der Heyden y Harvey (2012) concluyen queestas técnicas y programas, deben diseñarse sobre la base de resultados de indagación científica, con el fin de instalar procesos de toma de decisiones basados en evidencia que permitan un ajuste local de estas decisiones. El circuito de acciones en convivencia diseñadas sobre la base de la evidencia científica local, y evaluada en su eficacia y proceso de forma permanente en el tiempo, es definido en la literatura internacional, como un sistema de monitoreo de la convivencia escolar (Benbenishty \& Astor, 2011).

\section{Sistema de Monitoreo PACES-PUCV}

Durante los años 2013 y 2014, y en el marco de las acciones lideradas por el Programa de apoyo a la conviven- cia escolar, de la Pontificia Universidad Católica de Valparaíso PACES-PUCV, se llevó a cabo el proyecto "Diseño, desarrollo y validación de un sistema de monitoreo de la convivencia escolar", con el fin de permitir a las 44 escuelas primarias públicas de una comuna de la Quinta Región en Chile, conocer su estado actual y recibir apoyo para la toma de decisiones informada y orientada al mejoramiento escolar. Este sistema, fue desarrollado bajo un modelo de abajo-arriba o bottom-up, construido desde las bases (grass root movements), es decir, auto-gestadas, pertinentes a la realidad de cada escuela, y con ciertos recursos propios de la comunidad, fomentando así su resiliencia y empoderamiento (Hill \& Hupe, 2002; Sabatier, 1986), a la vez que buscó focalizarse en una visión institucional compartida (Catalán-Ahumada y Castro, 2016). Desde la perspectiva de diversos autores (Bolívar 1993; Domingo-Segovia, 2010), se trataría de una visión estratégica global, con perspectiva sistémica, que busca transitar desde y hacia todos los niveles de una escuela. Este sistema de monitoreo contempla la medición-evaluación de la convivencia, la devolución de resultados en un reporte de fácil interpretación on line y descargable, y asesorías para el diseño de acciones a nivel de gestión en convivencia escolar. Se evalúay asesora en dimensiones de clima escolar, victimización de estudiantes, victimización de personal del establecimiento, intimidación a profesores, peligrosidad en la escuela, contribución a la escuela, y satisfacción con la vida (López \& cols., 2013).

\section{Asesoramiento en convivencia escolar}

Las asesorías para la toma de decisiones basadas en los resultados de la medición-evaluación, implicó un seminario para apoyar la interpretación de los datos, y diversas acciones de acompañamiento según la modalidad en la cual se ubicaba cada escuela. Del total de las 44 escuelas, 15 escuelas fueron asesoradas en la modalidad "vía coaching"; 15 escuelas, en vía "web" y 14 escuelas, en vía "comunidades de aprendizaje". En todas las modalidades se constituyeron equipos de convivencia escolar compuestos por el Director o Directora, Encargado de Convivencia Escolar ${ }^{1}$, y dos o tres personas que trabajaban en cada escuela, ya fueran profesionales o personal de apoyo. Los supuestos que guiaron a las tres modalidades incluyeron de forma transversal las nociones de aprendizaje colectivo y conocimiento situado (Imbernón, 2008; Montecinos, 2003), ubicando a los asesores comocolegas críticos (Domingo-Segovia, 2003). La tabla 1 resume la modalidad de cada vía de asesoramiento:

Finalmente, se realizaron reuniones de trabajo entre un asesor y el equipo de convivencia escolar de cada escuela participante, presencialmente en cada establecimiento educativo.

1 En el marco de la actual Política Nacional de Violencia Escolar, y de la Ley de Violencia Escolar, cada establecimiento educativo del país debe nombrar un Encargado de Convivencia Escolar, quien será el responsable de gestionar las acciones en convivencia escolar dentro de cada escuela. 
Tabla 1. Modalidades de asesoramiento.

\begin{tabular}{|c|c|c|}
\hline Vía & Descripción & $\begin{array}{l}\text { Autores que inspiran la } \\
\text { propuesta }\end{array}$ \\
\hline Web & $\begin{array}{l}\text { Plataforma Moodle con lecturas, videos y actividades e } \\
\text { learning, con el fin de compartir experiencias de bienestar } \\
\text { psicosocial entre escuelas }\end{array}$ & $\begin{array}{l}\text { Kerr, Valois, Huebner, \& } \\
\text { Drane, 2011; Keyes } 1998\end{array}$ \\
\hline $\begin{array}{l}\text { Comunidades de } \\
\text { aprendizaje }\end{array}$ & $\begin{array}{l}\text { Sesiones de trabajo con grupos de escuelas, de } \\
\text { sistematización de experiencias exitosas }\end{array}$ & Lave \& Wenger, 1991 \\
\hline Coaching & $\begin{array}{l}\text { Sesiones de trabajo con grupos de escuelas, orientadas a } \\
\text { visualizar la "perspectiva del observador" }\end{array}$ & Echeverría, 2003 \\
\hline
\end{tabular}

Fuente: Elaboración propia.

Tabla 2. Muestreo y técnicas de recopilación de información.

\begin{tabular}{|c|c|c|c|c|}
\hline Escuela & Entrevistado/a(s) & $\begin{array}{l}\text { Tipo de } \\
\text { entrevista }\end{array}$ & $\begin{array}{l}\text { Modalidad de } \\
\text { asesoramiento }\end{array}$ & $\begin{array}{l}\text { Nivel de } \\
\text { adherencia }\end{array}$ \\
\hline Holanda & Encargado de convivencia. & Individual & Vía web & Bajo \\
\hline Ernesto Sábato & Encargada de convivencia & Individual & Vía Web & Bajo \\
\hline Salvador Allende & $\begin{array}{l}\text { Director, encargada de } \\
\text { convivencia }\end{array}$ & Grupal & Vía web & Medio \\
\hline Sudáfrica & $\begin{array}{l}\text { Directora, encargada de } \\
\text { convivencia }\end{array}$ & Grupal & Vía web & Medio \\
\hline O’higgins & Encargada de convivencia & $\begin{array}{l}2 \text { entrevistas } \\
\text { individuales }\end{array}$ & Vía web & Medio \\
\hline Gabriela Mistral & Encargada de convivencia & Individual & Vía web & Medio \\
\hline Pedro Lemebel & Encargada de convivencia & Individual & Vía Web & Alto \\
\hline Nicanor Parra & Directora (dos entrevistas) & Individual & $\begin{array}{l}\text { Comunidades de } \\
\text { aprendizaje }\end{array}$ & Medio \\
\hline Europa & $\begin{array}{l}\text { Directora, encargada de } \\
\text { convivencia, orientadora familiar, } \\
\text { profesora }\end{array}$ & Grupal & $\begin{array}{l}\text { Comunidades de } \\
\text { aprendizaje }\end{array}$ & Alto \\
\hline Bs. Aires & $\begin{array}{l}\text { Director, encargada de } \\
\text { convivencia }\end{array}$ & Grupal & $\begin{array}{l}\text { Comunidades de } \\
\text { aprendizaje }\end{array}$ & Alto \\
\hline García Márquez & $\begin{array}{l}\text { Psicólogo, Trabajadora Social, } 2 \\
\text { profesoras }\end{array}$ & Grupal & $\begin{array}{l}\text { Comunidades de } \\
\text { aprendizaje }\end{array}$ & Alto \\
\hline Vicente Huidobro & $\begin{array}{l}\text { Director, encargado de } \\
\text { convivencia }\end{array}$ & Grupal & Víacoaching & Medio \\
\hline Dr. Del Rio & $\begin{array}{l}\text { Director, encargado de } \\
\text { convivencia }\end{array}$ & $\begin{array}{l}2 \text { entrevistas } \\
\text { individuales }\end{array}$ & Víacoaching & Alto \\
\hline De Rokha & Encargada de convivencia & Individual & Víacoaching & Alto \\
\hline Eusebio Lillo & $\begin{array}{l}\text { Directora, Inspectora, } \\
\text { Orientadora, Jefe de UTP, } \\
\text { encargado de convivencia, } \\
\text { psicólogo. }\end{array}$ & Grupal & Víacoaching & Alto \\
\hline Sostenedor & $\begin{array}{l}\text { Encargada Comunal de } \\
\text { Convivencia Escolar }\end{array}$ & Individual & Gestión comunal & Alto \\
\hline
\end{tabular}

Fuente: Elaboración propia.

Sobre la base de todo lo anterior, es posible señalar que el sistema de monitoreo de la convivencia escolar implementado, posee un carácter innovador y peculiar, en cuanto si bienexiste un modelo de asesoría a la base, éste constituye un conjunto de medidas de apoyo flexibles, adaptadas a cada realidad, y por lo mismo, el impacto debe ser evaluado en diferentes niveles. En este contexto, se vuelve necesario conocer los significados construidos por el conjunto de usuarios del sistema de monitoreo, con el fin de mejorar acciones de este tipo. ¿Cómo evalúan los usuarios del sistema de monitoreo de la convivencia escolar? ¿Qué niveles de transferencia a sus prácticas cotidianas y de gestión, consideran que ha sido posible a partir de este sistema de monitoreo? ¿Existe algún cambio de significados en torno a la convivencia escolar, reconocido por los usuarios, a partir de esta experiencia? El presente estudio buscó dar respuesta a estas interrogantes, a través de una evaluación cualitativa en torno al sistema de monitoreo de la conviven- 
cia escolar implementado por el programa PACES-PUCV en una comuna de la Quinta Región en Chile.

\section{Método}

Diseño: El presente estudio muestra los resultados de un proceso de evaluación cualitativa (Di Virgilio \& Solano, 2012; Nirenberg, 2009) orientada a evaluar el logro de los objetivos del sistema de monitoreo de la convivencia escolar desde los significados de los usuarios, ya que se trata de un programa que puede ser mejorado, a través de recomendaciones orientadas a ajustar la acción a partir de la perspectiva de quienes protagonizan las acciones.

Muestreo y participantes: La selección de los participantes se basó en el criterio de adecuación y variabilidad (Quintana, 2006). Las escuelas participantes pertenecieron a diferentes modalidades de asesoramiento y niveles de adherencia al proceso (alta, media, baja). Ésta se midió a través de la asistencia a las diferentes instancias de asesoramiento bajo el siguiente criterio: alta- $71 \%$ a $100 \%$ - media $-41 \%$ a $70 \%$ - baja - $10 \%$ a $40 \%$. Al interior de cada escuela, se entrevistaron directores y equipos de convivencia escolar. Participó un total de 15 escuelas, a través de 17 entrevistas, algunas individuales y otras grupales (ver Tabla 2), contando además con una entrevista a la coordinadora comunal de convivencia escolar.

Técnicas de recopilación y análisis de información: Se realizaron entrevistas abiertas (Canales, 2006) individuales y grupales, dependiendo de la disponibilidad de cada escuela. Los temas tratados fueron: (i) Apreciación general sobre el proyecto, (ii) expectativas y percepciones sobre el proceso de aplicación de encuestas, reportes, y sobre los asesoramientos; (iii) transferencia del proyecto, (iv)posibilidades de tomar decisiones sobre la base del proyecto. Para el análisis se realizó un análisis de contenido (Cáceres, 2003). Las categorías fueron construidas de acuerdo con los objetivos del proyecto, así como con las fases del mismo. Los resultados de este artículo, presentan los significados construidos respecto de los aspectos centrales del proyecto, mientras que en las discusiones, se informa explícitamente en relación con los objetivos y componentes cualitativos que contempló el proyecto (ver Tabla 3), evaluando necesidades de mejoramiento para programas de este tipo o futuras versiones de este programa.

\section{Objetivos}

Procedimiento: Un equipo externo al equipo ejecutor del programa, se contactó con los directores de las escuelas. De un total de 18 escuelas seleccionadas, se logró concretar entrevistas con 15 escuelas. Las entrevistas fueron grabadas y transcritas, manteniendo criterios de confidencialidad respecto del nombre de las personas, así como de las escuelas. Por ello, los nombres presentados en este artículo no corresponden a la realidad.

\section{Resultados}

Los resultados se presentan de acuerdo a las categorías que emergieron del análisis de contenido.

\section{Contar con apoyos para tomar decisiones basadas en evidencia}

Los participantes evalúan de forma positiva la recogida de información que permite toma de decisiones basadas en evidencias, con el fin de complejizar la mirada que las escuelas estaban desarrollando de sí mismas. Lo que lo que más destaca de todo el proceso es el instrumento y su potencial de entrega de información válida. Este sentido sistémico, implica la necesidad de tomar decisiones cuyas evidencias impacten en algún nivel de problematización de los supuestos docentes, con el fin de comprender la interacción de diversos estamentos en el contexto escolar. De este modo, la encuesta y reportes, son vinculados con la posibilidad de ofrecer información que abarca diversos niveles de la escuela, incorporando además el contexto social.

Tabla 3. Relación de objetivos con componentes.

\begin{tabular}{ll}
\hline \multicolumn{1}{c}{ Objetivos } & \multicolumn{1}{c}{ Componentes } \\
\hline $\begin{array}{l}\text { Diseñar, desarrollar y validar un sistema de medición de violencia y clima } \\
\text { escolar que entregue información situada, específica y actualizada a los } \\
\text { establecimientos educacionales y al sostenedor. }\end{array}$ & $\begin{array}{l}\text { Medición de la Violencia y del } \\
\text { clima escolar }\end{array}$ \\
$\begin{array}{l}\text { Diseñar, desarrollar y validar un sistema de reporte digital que sea } \\
\text { comprensible e interpretable por la comunidad escolar. }\end{array}$ & Reporte con Soporte Digital \\
$\begin{array}{l}\text { Diseñar, desarrollar y validar tres sistemas de asesoramiento para la } \\
\text { facilitación del cambio en violencia y clima escolar: presencial, no-presencial } \\
\text { y en redes inter-escuelas. }\end{array}$ & $\begin{array}{l}\text { Asesoramiento Vía Web, Vía } \\
\text { Comunidades De Aprendizaje, } \\
\text { Vía Coaching }\end{array}$ \\
\hline
\end{tabular}

Fuente: Elaboración propia. 
...una de las cosas que nosotros necesitábamos era hablar de convivencia pero a partir de hechos reales y recogiendo información de actores importantes como son los alumnos, los profesores, los apoderados. Entonces ya no hablábamos sobre supuestos, sino que realmente nos dio una información, así bien, lo más objetiva dentro de lo posible, para poder nosotros considerar eso como un punto para el proyecto futuro, y para el trabajo que se quiera desarrollar, entonces es tener información significativa, válida para desde ahí levantar ¿no es cierto? las propuestas de trabajo. (Escuela Buenos Aires)

Es como una lógica que es mucho más social y no una perspectiva tan de la escuela, y eso les llamó mucho la atención. (Coordinadora Comunal)

El apoyo en la toma de decisiones, se evalúa como oportuno y pertinente debido a la ausencia de claridad que hoy tendrían las escuelas en Chile para la interpretación de políticas en convivencia escolar. Para las escuelas entrevistadas, el proyecto completa un vacío de información, en un contexto de incipientes políticas educativas que ofrecen escasos apoyos para su implementación. Los apoyos son valorados cuando éstos son contextualizados, destacando la necesidad de colocar el contexto local-institucional incluso por sobre la propia política.

...el proyecto vino a llenar una necesidad que había en ese momento, ¿ya? porque estaba surgiendo todo el tema del encargado de convivencia, súper incipiente. (Escuela Europa)

...yo creo que fue oportuna porque el tema de la convivenciase ha instalado en las escuelas como una necesidad de abordar, como un plan, como un programa. (Escuela Salvador Allende)

Los apoyos entregados, son valorados como un insumo efectivoque ofrece información diagnóstica. Esto se vuelve relevante, ya que se valora la recogida y análisis de información porque permite el mejoramiento escolar. Entre las dimensiones evaluadas, destacan para las escuelas especialmente el clima escolar.

Si yo creo que en la escuela la contribución directamente al fortalecimiento de la convivencia de manera de poder detectar o también hacer un levantamiento de necesidades en base de los resultados obtenidos por esta encuesta de este instrumento más que nada...Es más como un apoyo ¿ya? Se ve más como de apoyo y yo creo que llego en buen momento porque la verdad es que las preocupaciones en estos instantes justamente es el trato del alumno dentro del aula el trato entre ellos. El trato hacia los profesores, el trato también entre colegas, en fin, o sea es más que nada la dinámica de todos los estamentos de la institución porque para que realmente se vea que hay y que existe un buen trato. (Escuela De Rokha)
De todos modos, el potencial de usabilidad de la información entregada para la toma de decisiones a nivelinstitucional, dependía en parte de la capacidad de la propia escuela de construir políticas internas de gestión en convivencia escolar. En este sentido, hubo diferentesimpactos del proyecto, en escuelas que contaban con lineamientos institucionales previos en convivencia escolar, en comparación con escuelas que no contaban con éstos.

no hemos podido hacer es ese cruce de información especifico de los resultados que se obtuvo con la encuesta, y transformarla todavía en actos, porque siempre hemos tenido que estar generando como te decía, procesos que no están instalados en el colegio, en el fondo política de convivencia, política de orientación, que no existían. (Escuela Vicente Huidobro)

En términos generales el proyecto presenta algunos desafíos que son expresados en las entrevistas. Algunos colegios esperaban mayor presencia de parte de los asesores, lo que podría vincularse con escuelas que aún no tienen instalados procesos autónomos de mejoramiento escolar.

Lo que pasa que en ese particularmente el tema de la devuelta de información que ellos deberian haber venido a la escuela, ellos deberían habernos presentado la cifra, los análisis que han hecho, eso en particular. (Escuela O'higgins)

Finalmente, un elemento que impacta de forma negativa en la transferencia del proyecto, se vincula con las condiciones de trabajo en las escuelas, ya que la rotación del personal influyó de forma directa en las posibilidades de proyectar el trabajo en convivencia escolar, así como en gestión en general.

Lo que pasa es que en ese momento estaba a cargo una profesora de la convivencia escolar, profesora que ya no está en la escuela...no se sociabilizó mucho el trabajo aquí dentro del establecimiento. (Escuela Holanda)

\section{El papel de los componentes del proyecto, en la toma de decisiones efectivas: encuesta y reportes}

El primer componente del sistema de monitoreo de la convivencia escolar implementado por este equipo, es una encuesta cuyos resultados buscaron impactar en el (re)diseño de políticas internas en cada escuela, así como a nivel comunal. Esta encuesta, es evaluada por las escuelas como un instrumento complejo y relevante, aunque en el proceso de aplicación, hubo dificultades de tipo operativas.

...fue interesante la encuesta en el sentido que era muy completa, abarcaba diferentes temáticas y no sólo desde una mirada desde el déficit...lo más complejo fue el uso de las claves, que eso si era como... pero es más bien 
me imagino como un tema de programación informática. (Escuela Europa)

Los colegios señalan que el papel de esta encuesta para tomar decisiones, fue algo que quedó claro cuando los asesores llegan a la escuela a explicar en detalle la encuesta, así como el proceso de aplicación. Esta instancia aparece como una oportunidad de negociación de significados, y por lo tanto, se vislumbra como un necesario espacio para el compromiso de la escuela. Finalmente, la encuesta destaca por su potencial de entrega de información que pone en el centro a los protagonistas de la vida escolar.

...Ya cuando vino Rosa-refiriéndose a la coordinadora del proyecto-, ya el tema se disipó un poco más, ya hubo mayor claridad. (Escuela Pedro Lemebel)

...entender que el profesor, los alumnos, los lugares, los padres, las perspectivas de 'los niños piensan esto de mí y yo no sabia', porque normalmente están en la visión de 'yo no pienso esto de los niños. Los niños son malos, los niños son desordenados, los niños son mal educados, los niños son...' y no reciben la devuelta, y esa perspectiva también Ilama mucho la atención. (Coordinadora Comunal)

El segundo componente del sistema de monitoreo de la convivencia escolar, es el reporte. El reporte se devuelve de forma on line, permitiendo a cada escuela compararse con la comuna. Lo primero que se evalúa de forma positiva, es la claridad del reporte, así como de la tabulación de datos a través de gráficos simples. La tendencia en las escuelas, fue referirse al reporte en vinculación con la encuesta, evidenciando una comprensión articulada entre ambos componentes.

\footnotetext{
... el reporte se entiende, es de fácil acceso, nos dieron una página para ingresar, una clave para ingresar. (Escuela Dr. Del Río)
}

Presentar los resultados en un reporte que informara en términos globales, y luego por ítem, facilitó la transferencia de estos resultados desde el equipo de gestión escolar a los profesores, destacando en todos los casos lo relevante de la socialización de estos datos con el fin de problematizar concepciones instaladas en las escuelas. Estos reportes facilitaron que la evidencia fuera considerada como necesaria para tomar decisiones.

Cuando llegaron los resultados de este diagnóstico se sociabilizó con los profesores, con ello, se sociabilizo por intermedio de intervención pedagógica enfocado a la convivencia, y realmente quedamos sorprendidos en algunos rasgos, en algunas percepciones de los alumnos en relación a la convivencia, en el trato de ellos, entre los alumnos, que no eran percibidos por los profesores. (Escuela Nicanor Parra)
De todas las dimensiones evaluadas y asesoradas clima escolar, victimización de estudiantes, victimización de personal del establecimiento, intimidación a profesores, peligrosidad en la escuela, contribución a la escuela, y satisfacción con la vida- las más destacadas como relevantes para el diseño de acciones, se vinculan con la relación entre los propios estudiantes y la contribución a la escuela.

\section{El sistema de apoyo: las modalidades de asesoramientos}

Los equipos de convivencia escolar, participaron de tres modalidades de asesoramiento: vía web, comunidades de aprendizaje y vía coaching. En el primer caso, algunas escuelas se inscribieron pensando que en esta modalidad debían trabajar menos. Esta decisión fue tomada, ya sea porque el proyecto no les hacía mucho sentido, o ya sea porque expresaban altos niveles de intensificación de sus trabajos, con escasa posibilidad de salir del colegio a encuentros presenciales.

Algunas escuelas que se inscriben en esta modalidad, señalan que no contaron con suficiente información para elegir. La tendencia fue que las escuelas que seleccionan vía web, tuvieron niveles de adherencia medios o bajos en relación con el proyecto. La única escuela con una alta adherencia en esta modalidad, señala que los aprendizajes desarrollados lograron impactar en las políticas institucionales, aunque confirma la baja adherencia.

...o sea cuando se presentó la hoja, era, una hojita así no
más donde tú marcabas ahí.(Escuela Sudáfrica)

Sí, mucho de lo que había en el asesoramiento lo apliqué, lo aplicamos, perdón, en el plan de convivencia escolar, en el desarrollo desde los textos que se nos entregaban...Eran poquitas las escuelas que participábamos activamente en la modalidad vía web. (Escuela Pedro Lemebel)

Respecto de la modalidad vía coaching, las escuelas optaron a través deargumentosestratégicos, destacando el liderazgo del Director. Estas escuelas consideraron que la asesoría ofrecía la posibilidad deconstruir prácticas innovadoras, destacando por un alto impacto en las concepciones docentes.

...igual la modalidad del coaching es la más, yo creo que es lo que más provoca impacto cuando se necesita girar un poco y movilizar a la gente pa' mirar desde otro lado. (Director, Escuela Dr. Del Río)

Por su lado, en la modalidad de comunidades de aprendizaje, lo que más se destacó fue la posibilidad de conocer el trabajo de otros colegios y así desarrollar algún nivel de articulación entre las escuelas. El espacio fue especialmente valorado, por considerar que instancias de este tipo son poco frecuentes. 
... tomamos la opción de juntarnos con otras escuelas y de alguna manera ver lo que nosotros como escuela estábamos realizando y lo que las otras escuelas también estaban aplicando eso eh... lo consideramos una fortaleza, ya que no hay muchas instancias en que uno como escuela pueda compartir con otras escuelas y pueda ver qué es lo que está sucediendo. (Escuela Buenos Aires)

Finalmente, cabe destacar que desde la perspectiva comunal, el valor del proyecto se sitúa en cómo el programa permitió evaluar y retroalimentar sistémicamente las escuelas, así como apoyar en los procesos de implementación de una política nacional que muchas veces no es comprendida por los colegios.

Les ayudaron a trabajar con la información. Les ayudaron a entender parte de procesos internos que ellos no entendían, si esto es todo nuevo para nosotros. Entonces a entender una lógica que esté como un poco fuera de lo que es el sistema educacional chileno, no sé cómo explicarlo. (Coordinadora Comunal)

\section{Discusiones y conclusiones}

Dentro de los componentes de este sistema, laevaluación-medición de la convivencia escolar destaca como un insumo pertinente en el actual contexto nacional, valorándose el papel de la evidencia (Van Der Heyden \& Harvey, 2012) que efectivamente permita tomar decisiones que conduzcan al centro educativo en la gestión de sus políticas internas (Justice, 2006; Klinger \& Edwards, 2006), así como a dialogar con el contexto político más amplio. En este sentido, las escuelas que participan del sistema de monitoreo, colocan en el centro de la mejora escolar a la propia escuela, destacando que la efectividad sólo es posible si la priorización de necesidades surge del propio colegio (Astor \& cols., 2004).

En este contexto, el componente de los reportes permite a las escuelas recibir una devolución de información que instala procesos de decisiones basados en evidencia con un ajuste local de éstas (Benbenishty \& Astor, 2011), y el formato de devolución de gráficos resulta simple de ser comprendido. Esto facilita la reflexión y el debate dentro de las escuelas, con el fin de permitir la profundización en temas prioritarios para el colegio y así elaborar estrategias de mejora con carácter colectivo y participativo (Imbernón, 2008).

Se valora la mirada externa y el análisis que invitó a una reflexión interna en cada escuela, permitiendo que los colegios utilizaran la información como un apoyo a sus procesos de autoevaluación más que de inspección (Domingo-Segovia, 2010). Sin embargo, el componente de interpretación de los reportes requiere mayor presencia de los equipos ejecutores del proyecto dentro de las escuelas. En este sentido, si bien el proyecto se funda desde la consideración de las escuelas como espacios autónomos, estos resultados muestran que la valoración de los conocimientos que los propios profesores y profesionales de las escuelas han adquirido en su experiencia (Montecinos, 2003), debe ser mediada y acompañada para que tenga incidencia en la gestión del centro educativo, en este caso, de la convivencia escolar. El reconocimiento de la autonomía de los centros educativos, no debe ser interpretado necesariamente como la independencia de los mismos para analizar resultados de evaluaciones de las cuales participan, y el proceso de acompañamiento debiese considerar la necesaria mediación e interpretación de datos. Lo anterior, implica que un sistema de monitoreo de estas características, debe considerar el componente de interpretación de los reportes como parte del sistema de apoyo, lo que da inicio a la fase de asesoramiento no es el trabajo específico de planificación de cada modalidad (web, coaching y comunidades de aprendizaje), sino más bien, la devolución de resultados y las maneras en que metodológicamente se decide devolver.

En cuanto al componente de modalidades de asesoramiento, lo que más destaca es el énfasis en el aprendizaje entre escuelas, destacando cómo el intercambio de experiencias con colegas de otros colegios, permitió que se formaran alianzas, dando cuenta de un proceso de construcción de comunidades de aprendizaje (Lave \& Wenger, 1991). Sin embargo, la modalidad vía web, constituye una excepción, ya que una débil gestión de las escuelas que eligieron esta opción, sumado a la intensificación del trabajo dificultó una mejor y mayor dedicación a la plataforma moodle, a la vez que inhibió el trabajo articulado entre diferentes escuelas. Finalmente, más allá de las posibilidades de mejoramiento y ajuste que un sistema de este tipo requiere, resultan interesantes los significados y concepciones que evolucionan durante la implementación de este sistema. La sensibilización del modelo ecológico que subyace a esta propuesta (Astor \& Benbenishty, 2006) así como el carácter sistémico de la misma (Domingo-Segovia, 2010), son aprehendidos por los equipos de convivencia cuyos análisisdejan en evidencia la importancia de que todos los actores y estamentos estén articulados por una meta común, reconociendo la participación estudiantil, así como el necesario liderazgo del director (Bolívar \& cols., 2013) en la conducción de actividades y lineamientos estratégicos de mejoramiento de la convivencia escolar.

De todos modos, siguen primando significados sobre convivencia escolar fundamentalmente asociados a la interacción de estudiantes. Esto explicaría por qué los resultados mostraron que, por parte de las escuelas, el interés y énfasis estuvo puesto fundamentalmente en las encuestas y reportes de los estudiantes, y no así en las de profesores y apoderados. En este sentido, sería relevante investigar sobre el papel de otros actores educativos en las definiciones sobre convivencia escolar que ofrecen los centros educativos, así como la valoración que las escuelas asignan a éstos para las transformaciones en convivencia escolar. 


\section{Referencias}

Abad, M. (2002). Las políticas de juventud desde la perspectiva de la relación entre convivencia, ciudadanía y nueva condición juvenil. Ultima década, 10 (16), 117-152.http://dx.doi.org/10.4067/ S0718-22362002000100005

Agencia de Calidad de la educación [ACE] (2015). Calidad escolar desde la percepción de los actores claves del sistema: Un estudio cualitativo en educación básica. Disponível: http://archivos. agenciaeducacion.cl/calidad_escolar_desde_la_percepcion_de_ los_actores_clave_del_sistema.pdf

Astor, R. A.; Benbenishty, R.; Meyer, H. A. (2004). Monitoring and mapping student victimization in schools. Theory into practice, 43 (1), 39-49. http://dx.doi.org/10.1207/s15430421tip4301_6

Astor, R. A.; Benbenishty, R. (2006). Zero tolerance for zero knowledge: Empowering schools and communities with data and democracy. Los Ángeles: University of Southern California, Urban Policy Brief, Urban Initiative.

Benbenishty, R.; Astor, R.A. (2005). School Violence in Context: Culture, Neighborhood, Family, School, and Gender. Nueva York: Oxford University.

Benbenishty, R.; Astor, R. A. (2011). Making the case for an international perspective on school violence: Implications for theory, research, policy, and assessment. In: Jimerson, S. R.; Nickerson, A. B.; Mayer, M. J.; Furlong, M. J. (Orgs.), The handbook of school violence and school safety: International research and practice (pp. 18-36). Nueva York: Routldege.

Bender Sebring, P.; Montgomery, N. (2014). Los cinco apoyos esenciales para el mejoramiento de los aprendizajes en la escuela: movilizando los resultados. Pensamiento Educativo, 51(1), 66-85. http://dx.doi.org/10.7764/PEL.51.1.2014.19

Bolivar, A. (1993). Cambio educativo y cultura escolar: resistencia y reconstrucción. Innovación educativa, (2) 13-22. Recuperado: 7 sept. 2010. Disponível: https://dspace.usc.es/ bitstream/10347/5317/1/pg_015-024_inneduc2.pdf

Bolívar, A. (2010). El liderazgo educativo y su papel en la mejora: una revisión actual de sus posibilidades y limitaciones. Psicoperspectivas, 9(2), 9-33.http://dx.doi. org/10.5027/psicoperspectivas-Vol9-Issue2-fulltext-112

Bolívar, A.; López, J.; Murillo, F. J. (2013). Liderazgo en las instituciones educativas. Una revisión de líneas de investigación. Revista Fuentes, (14), 15-60. Recuperado: 4 abr. 2015. Disponível: http://institucional.us.es/revistas/fuente/14/Firma\%20invitada.pdf

Cáceres, P. (2003). Análisis cualitativo de contenido: una alternativa metodológica alcanzable. Psicoperspectivas, 2(1), 53-82. Recuperado: 7 mar. 2011. Disponível: http://www. psicoperspectivas.cl/index.php/psicoperspectivas/article/
viewFile/3/3

Catalán-Ahumada, J.; Castro, P. J. (2016). Reflexión colectiva sistemática: un estudio orientado al desarrollo profesional docente. Psicología Escolar e Educacional, 20(1),157-167.http:// dx.doi.org/10.1590/2175-3539/2015/0201949

Canales C, M. (2006). Metodologías de investigación social: Introducción a los oficios. Santiago: LOM

Di Virgilio, M. M.; Solano, R. (2012). Monitoreo y evaluación de políticas, programas y proyectos sociales. Bs. Aires: CIPPEC.

Domingo-Segovia, J. (2010). Comprender y redireccionar las prácticas de asesoría. Revista Iberoamericana de educación, (54), 65-83. Recuperado: 7 mar. 2011. Disponível: http://www.rieoei.org/ rie54a03.pdf

Echeverría, R. (2003). Ontología del lenguaje. Santiago: JC Sáez.

Espinoza, E. (2006). Impacto del maltrato escolar en el rendimiento académico. Revista electronica de investigación psicoeducativa, 4(9), 221-238. Recuperado: 10 oct. 2011. Disponível: http://www.investigacion-psicopedagogica.org/revista/ articulos/9/espannol/Art_9_64.pdf

Furlong, M.; Greif, J. L.; Bates, M. P.; Whipple, A. D.; Jimenez, T. C.; Morrison, R. (2005). Development of the California school climate and safety survey short form. Psychology in the schools, 42137 149.http://dx.doi.org/10.1002/pits.20053

Giraldo, L. D.; Mera, R. (2014). Clima social escolar: percepción del estudiante. Colombia Médica, 31 (1) 23-27. Recuperado: 5 may. 2016. Disponível: http://colombiamedica.univalle.edu.co/index. php/comedica/article/view/148/150

Hill, M. J.; Hupe, P. L. (2002). Implementing public policy: Governance in Theory and Practice.London: Sage Publications.

Imbernón, F. (2008). Asesorar o Dirigir. El Papel del Asesor/a Colaborativo en una Formación Permanente Centrada en el Profesorado y en el Contexto. REICE, 5(1), 145-152. Recuperado: 3 mar. 2009. Disponível: http://www.redalyc.org/pdf/551/55100108. pdf

Justice, L. (2006). Evidence-Based Practice, Response to Intervention, and the Prevention of Reading Difficulties. Language, Speech, and Hearing Services in Schools, 37, 284-297.doi:10.1044/01611461(2006/033)

Kerr, J.C.; Valois, R. F.; Huebner, E. S.; Drane, J. W. (2011). Life satisfaction and peer victimization among USA public high school adolescents. Child Ind Res, 4(1), 127-144. http://dx.doi. org/10.1007/s12187-010-9078-y

Keyes, C. L. M. (1998). Social well-being.Social Psychology Quarterly, 61(2), 121-140. 
Klinger, J. A.; Edwards, P. A. (2006).Cultural Considerations with Response to Intervention Models.Reading Research Quarterly, 41(1), 108-117 http://dx.doi.org/10.1598/RRQ.41.1.6

Lave, J.; Wenger, E. (1991). Situated learning: Legitimate peripheral participation.Cambridge: Cambridge University.

López, V.; Ascorra, P.; Bilbao, M.; Carrasco, C.; Morales, M.; Villalobos, B.; Ayala, Á. (2013). Monitorear la convivencia escolar para fortalecer (no disminuir) las capacidades de las escuelas. RIEE, 6(2), 201-219. Recuperado: 3 ene. 2014. Disponível: http://www. rinace.net/riee/numeros/vol6-num2/art11.pdf

López, V.; Ascorra, P., Bilbao, M. A., Oyanedel, J.; Moya, I.; Morales, M. (2012). EL Efecto del Ambiente Escolar sobre losResultados PISA 2009. Informe final Fondo de Investigación y Desarrollo en Educación - FONIDE Departamento de Estudios y Desarrollo. División de Planificación y Presupuesto. Ministerio de Educación. Disponível: http://portales.mineduc.cl/usuarios/ acalidad/doc/201211291046160.El\%20Ambiente\%20Escolar\%20 Incide\%20en\%20los\%20Resultados\%20PISA.pdf

Magendzo K, A. (2007). Formación de estudiantes deliberantes para una democracia deliberativa. REICE, 5 (4). Recuperado: 5 jun. 2014. Disponível: http://www.redalyc.org/pdf/551/55140505.pdf

MINEDUC (2003). Política de Convivencia Escolar. Santiago: MINEDUC.

MINEDUC (2011).Política Nacional de Convivencia Escolar. Santiago: MINEDUC.

MINEDUC (2014). Estándares Indicativos de Desempeño para los Establecimientos Educacionales y sus Sostenedores. Santiago: MINEDUC.

MINEDUC (2015). Política Nacional de Convivencia Escolar. Santiago: MINEDUC.
Montecinos, C. (2003). Desarrollo profesional docente y aprendizaje colectivo. Psicoperspectivas, 2, 105-28. Recuperado: 10 jul. 2006. Disponível: http://www.psiucv.cl/wp-content/uploads/2012/11/ DESARROLLO-PROFESIONAL-DOCENT.pdf

Nirenberg, O. (2009). Evaluación y participación: orientaciones conceptuales para una mejora de la gestión. In: Chiara,M.; Di Virgilio,M. (Orgs.), Gestión de la política social. Conceptos y herramientas (pp. 273-306). Buenos Aires: Prometeo-UNGS.

Pérez, J. C.; Astudillo, J.; Varela, J.; Lecannelier, F. (2013). Evaluación de la efectividad del Programa Vínculos para la prevención e intervención del Bullying en Santiago de Chile. Psicologia Escolar e Educacional, 17(1), 163-72. http://dx.doi.org/10.1590/S141385572013000100017

Quintana, A. (2006). Metodología de investigación científica cualitativa. In: Quintana, A.; Montgomery,W. (Orgs.),Psicología: Tópicos de actualidad.(Pp.47-84)Lima: UNMSM.

Sabatier, P. A. (1986). Top-down and bottom-up approaches to implementation research: a critical analysis and suggested synthesis. Journal of public policy, 6(1), 21-48.http://dx.doi. org/10.1017/S0143814X00003846

Valdés, H.; Treviño, E.; Acevedo, C. G.; Castro, M.; Carrillo, S.; Costilla, R.; Bogoya, D.; Pardo, C. (2008). Los aprendizajes de losestudiantes de América Latina y el Caribe: Primer reporte de los resultados del Segundo Estudio Regional Comparativo y Explicativo. Disponível: http://unesdoc.unesco.org/images/0016/001606/160660s.pdf

Van Der Heyden, A.; Harvey, M. (2012). Using Data to Advance Learning Outcomes in Schools. Journal of Positive Behavior Interventions, 6(1), 21-48. doi: 10.1177/1098300712442387

Recebido em: 21 de outubro de 2016 Aprovado em: 17 de agosto de 2017

Investigación financiada por la Comisión Nacional de Investigación Científica y Tecnológica de Chile CONICYT a través de los proyectos FondefIT n ${ }^{\circ}$ 14i10132; Fondecyt $n^{\circ} 1140960$ y PIA Conicyt CIE 160009. unrestricted use, distribution and reproduction in any medium, provided the original article is properly cited. 